\title{
Depresión, ansiedad y salud autopercibida en estudiantes de Medicina: un estudio transversal.
}

\section{Depression, anxiety and self-perceived health in medical students: a cross-sectional study.}

\author{
Iván Gutiérrez Pastor ${ }^{1}$, José Antonio Quesada Rico ${ }^{2 *}$, Aarón Gutiérrez Pastor ${ }^{3}$, \\ Rauf Nouni García ${ }^{2}$, María Concepción Carratalá Munuera ${ }^{2}$
}

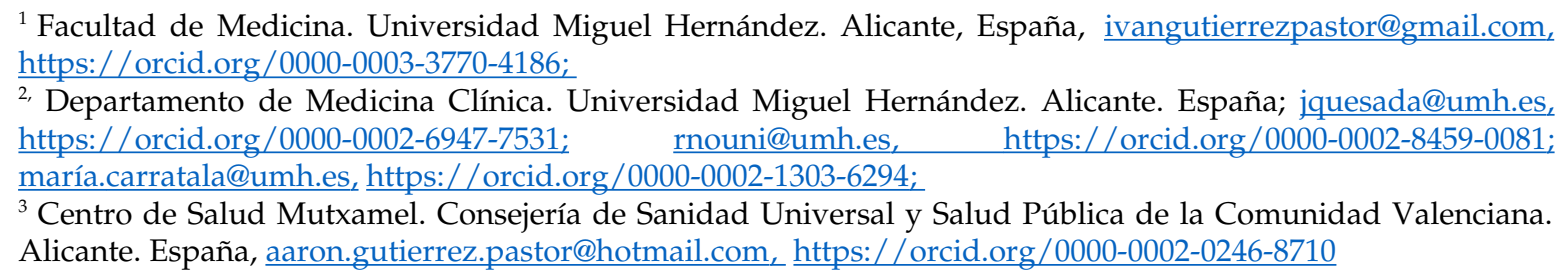

*Correspondencia: jquesada@umh.es

Recibido: 24-02-2021; Aceptado: 12-05-2021; Publicado: 17-05-2021

Resumen: la salud mental de estudiantes universitarios es un motivo de investigación a nivel internacional. El objetivo de este estudio fue estimar la prevalencia de ansiedad, depresión y estado de salud autopercibida de los estudiantes de medicina de la Universidad Miguel Hernández y analizar su asociación con diferentes variables sociodemográficas y del estilo de vida. Para ello, se realizó un estudio descriptivo transversal de estudiantes de medicina de $1^{\circ}$ a $6^{\circ}$ curso de la Universidad Miguel Hernández de Elche durante el curso 2019-20. Se utilizaron la Escala Visual Analógica (EVA) del European Quality of Life-5 Dimensions (EQ-5D), la Escala de Ansiedad y Depresión de Goldberg (EADG), el estudio Prevención con Dieta Mediterránea (PREDIMED), el Alcohol Use Disorders Identification Test (AUDIT-C) y el Test de Fagerström. Todos los cuestionarios están validados en España. Para el análisis se ajustaron modelos logísticos multivariantes. Fueron encuestados 474 estudiantes. La tasa de respuesta global fue del $55.26 \%$. Un $68.1 \%$ de la muestra eran mujeres. Se estimó una prevalencia de probable ansiedad del $54.9 \%$, probable depresión del $60.9 \%$ y probable ansiedad o depresión del $73.3 \%$. La salud autopercibida regular-mala-muy mala fue del $8.9 \%$. Se detectó una asociación estadísticamente significativa con el sexo, la edad, el curso académico, el municipio de residencia, el tipo de alimentación, la actividad física y el consumo de tóxicos. Como conclusión, los estudiantes de medicina de la Universidad Miguel Hernández presentaron una prevalencia alta de probable ansiedad y depresión. Se observaron diferencias en función del sexo, curso académico, tipo de alimentación y consumo de tóxicos.

Palabras clave: Estudiantes de medicina, Salud autopercibida, Estilo de vida, Dieta mediterránea, Consumo de tóxicos.

Abstract: the mental health of university students is a reason for international research. The objective of this study was to estimate the prevalence of anxiety, depression and self-perceived health status of medical students from the Miguel Hernandez University and analyze their association with different sociodemographic and lifestyle variables. We did a cross-sectional descriptive study of medical students from $1^{\text {st }}$ to $6^{\text {th }}$ year of the Miguel Hernandez University during the 2019-20 academic year. They were used the Visual Analogue Scale (VAS) of the European Quality of Life-5 Dimensions (EQ-5D), the Goldberg Anxiety and Depression Scale (GADS), the Mediterranean Diet Prevention study (PREDIMED), the Alcohol Use Disorders Identification Test (AUDIT-C) and the Fagerström Test were used. All the questionnaires are validated in Spain. For the analysis, multivariate logistic models were adjusted. They were 
surveyed 474 students. The overall response rate was $55.26 \% .68 .1 \%$ of the sample were women. A prevalence of probable anxiety of $54.9 \%$; probable depression of $60.9 \%$ and probable anxiety or depression of $73.3 \%$ was estimated. Regular-bad-very bad self-perceived health was only $8.9 \%$. A statistically significant association was detected between the aforementioned variables and sex, age, academic year, municipality of residence, type of diet, physical activity and consumption of toxics. The medical students of the Miguel Hernandez University presented a high prevalence of probable anxiety and depression. Differences were observed based on sex, academic year, type of diet and consumption of toxic drugs.

Keywords: Key words: Medical students, Self-perceived health, Lifestyle, Mediterranean diet, Consumption of toxics.

\section{Introducción}

Durante la etapa universitaria, los estudiantes desarrollan hábitos negativos para su salud (1). Esto favorece el aumento de morbilidad y mortalidad, motivado por las dietas poco saludables, la inactividad física y el consumo de alcohol y tabaco (2). En la última década, el patrón de dieta occidental, caracterizado por una alta ingesta de carne y grasas saturadas y un bajo consumo de frutas y verduras, ha sustituido al modelo de dieta mediterránea en universitarios españoles (3). Son ampliamente conocidos los beneficios de la dieta mediterránea en la prevención de enfermedades crónicas (4), especialmente entre los estudiantes de ciencias de la salud. Sin embargo, el estudio 'Dieta, Salud y Antropometría-Universidad Miguel Hernández' (DiSA-UMH) en 2016 detectó que solo un $14.4 \%$ de estos estudiantes presentaron una alta adherencia a la dieta mediterránea (5).

En cuanto al consumo de tóxicos, el tabaco y el alcohol suponen una importante amenaza para la salud pública mundial (6-7). El estudio DiSA-UMH en 2016 estimó que el $65.5 \%$ de los estudiantes de ciencias de la salud presentaron una baja ingesta diaria de alcohol, el 33.6\% moderada y el 0.9\% alta (8). Según el Instituto Nacional de Estadística de España (INE), el 27\% de adultos jóvenes españoles es fumador diario y el $4.5 \%$ es fumador ocasional (9). Sin embargo, en una revisión europea en estudiantes de medicina entre los años 1988-2013, la prevalencia fue del 19\% de fumadores (10).

Por otro lado, desde que la Organización Mundial de la Salud (OMS) definió el concepto de salud como «aquel estado de completo bienestar físico, mental y social, y no sólo la ausencia de enfermedad» (11), el estudio de los indicadores de salud se encuentra en auge y conceptos como 'calidad de vida', 'salud autopercibida', 'felicidad', 'salud mental' y 'bienestar social' son de uso común (12). La salud autopercibida es considerada uno de los más importantes. Representa la percepción individual y la evaluación de la propia salud y se ha estudiado frecuentemente su relación con el sexo, la situación demográfica y el estilo de vida, entre otros aspectos (13).

La salud mental de los estudiantes sanitarios es otro motivo importante de investigación. Dos revisiones en estudiantes de medicina estimaron que la prevalencia mundial de ansiedad era del 33.8\% (rango: 29.2\%-38.7\%) mientras que la de depresión o síntomas depresivos era del 27.2\% (rango: 9.3\%-55.9\%) (14-15). La depresión se ha relacionado con el abuso de sustancias, el agotamiento y los pensamientos suicidas en este grupo de población. Años después podría verse afectada la calidad asistencial de los pacientes debido a una peor comunicación, un mayor número de errores médicos y una falta de implicación asistencial por parte del médico residente (16). Por ello, es importante conocer el estado actual de la salud mental de los estudiantes de medicina con el fin de establecer futuras estrategias de intervención en educación para la salud. 
El objetivo de este trabajo fue estimar la prevalencia de ansiedad, depresión y estado de salud autopercibida de los estudiantes de medicina de la Universidad Miguel Hernández (UMH) y analizar su asociación con diferentes factores sociodemográficos y del estilo de vida.

\section{Métodos}

\subsection{Diseño}

Se realizó un estudio descriptivo transversal mediante cuestionarios validados que analizó el estado de salud autopercibida, la prevalencia de ansiedad y depresión y las características del estilo de vida (tipo de alimentación, actividad física y consumo de tóxicos) de los estudiantes de medicina de la UMH de España.

\subsection{Población}

La población del estudio estaba compuesta por los 855 estudiantes de medicina de primer a sexto año matriculados en la UMH durante el curso 2019-2020. Se incluyó a aquellos alumnos que aceptaron participar y cumplimentaron la encuesta de forma voluntaria. Se excluyeron a aquellos alumnos que no estuvieron presentes en el momento de pasar la encuesta o declinaron hacerlo.

\subsection{Muestreo}

Se utilizó un muestreo consecutivo. La recogida de datos se realizó en el periodo comprendido entre los meses de septiembre y noviembre de 2019, pasando las encuestas en las aulas de la UMH y durante el Congreso Nacional de Estudiantes de Medicina 2019 (CNEM) a un total de 474 estudiantes. Se informó del objetivo y metodología del estudio a los participantes y se mantuvo el anonimato de sus respuestas en todo momento.

\subsection{Variables respuesta}

- Salud autopercibida: se valoró mediante la Escala Visual Analógica (EVA) del European Quality of Life-5 Dimensions (EQ-5D), con una puntuación de 0 a 100 que va del peor al mejor estado de salud imaginable. El estudiante debe marcar su estado de salud global actual. Se establecieron dos grupos, un estado de salud autopercibido regular-malo-muy malo (valores 0-59) y un estado de salud autopercibido buenobueno (valores 60-100). Se recogió información sobre la percepción de salud actual comparada con la de hace 2 años mediante una pregunta extraída del cuestionario C4 del proyecto español 'Seguimiento Universidad de Navarra' (SUN).

- Depresión y ansiedad: se valoró mediante la Escala de Ansiedad y Depresión de Goldberg (EADG) que se estructura en dos sub-escalas (ansiedad y depresión) con 9 ítems de respuesta dicotómica cada una. Los 4 ítems iniciales indican la probabilidad de que exista ansiedad o depresión y los 5 ítems restantes se completan en caso de presentar $\geq 2$ respuestas positivas para ansiedad $o \geq 1$ respuestas positivas para depresión, con un total de 9 puntos independientes para cada sub-escala. Se propusieron como puntos de corte una puntuación $\geq 4$ para ansiedad y $\geq 2$ para depresión, con una sensibilidad del $83 \%$ y una especificidad del $82 \%$. 


\subsection{Variables de exposición}

- Factores sociodemográficos: se recogieron datos sobre sexo (hombre/mujer), edad (años), municipio de residencia, curso (primer a sexto curso), estudios previos (bachiller/otros) y trabajo actual (sí/no).

- Estilo de vida: se evaluó la adherencia a la dieta mediterránea mediante el cuestionario 'Prevención con Dieta Mediterránea' (PREDIMED) que consta de 14 ítems cuyas respuestas afirmativas suman 1 punto cada una. Se definió 'adherencia baja' una puntuación <9 y 'adherencia aceptable' una puntuación $\geq 9$. Se evaluaron otras características de la dieta del estudiante mediante una pregunta extraída del cuestionario C0 del proyecto SUN. Se establecieron 2 grupos entre los estudiantes que tenían una dieta especial, los que seguían un patrón de dieta vegetariana y los que seguían otras dietas. Se evaluó la actividad física mediante la pregunta 112 de la Encuesta Nacional de Salud de España 2017 (ENSE).

- Consumo de tóxicos: se evaluó el consumo de alcohol (preguntas A1-A2) y el consumo de cannabis (preguntas M1-M2) mediante la Encuesta sobre alcohol y otras drogas en España 2017 (EDADES). Se evaluó el consumo de tabaco mediante las preguntas 121122-124 de la ENSE. Se valoró si existe consumo de riesgo de alcohol, entre los estudiantes que beben, mediante el Alcohol Use Disorders Identification Test (AUDITC) basado en 3 ítems y una puntuación final de 0 a 12 puntos. Se consideró 'consumo de riesgo' una puntuación $\geq 4$ en hombres y $\geq 3$ en mujeres. Se evaluó la dependencia a la nicotina, entre los estudiantes que fuman a diario, mediante el Test de Fagerström, formado por 6 ítems con una puntuación final de 0 a 10 puntos. Se definió 'baja dependencia' una puntuación 0-4, 'dependencia media' una puntuación 5-6 y 'alta dependencia' una puntuación 7-10.

\subsection{Análisis estadístico}

Se realizó un análisis descriptivo de todas las variables mediante cálculo de frecuencias para las cualitativas y valores mínimo, máximo, medio y desviación estándar para las cuantitativas. Se analizaron los factores asociados a salud autopercibida, ansiedad, depresión y ansiedad o depresión mediante tablas de contingencia, aplicando el test ChiCuadrado para las variables cualitativas, y comparación de valores medios para las cuantitativas, aplicando el test $\mathrm{T}$ de Student. Para estimar las magnitudes de las asociaciones con presencia de las variables respuesta, se han ajustado modelos logísticos multivariantes. Se han estimado los Odds Ratios (OR), junto con sus intervalos de confianza al $95 \%$. Se ha realizado un procedimiento de selección de variables stepwise basado en el criterio AIC (Akaike Information Criterium). Se muestran indicadores de bondad de ajuste e indicadores predictivos como la curva ROC. Los análisis se realizaron mediante el programa SPSS v.26 y el programa R v.3.6.0.

\section{Resultados}

\subsection{Características sociodemográficas}

Un total de 473 encuestados conformaban el estudio. Un $68.1 \%$ de la muestra eran mujeres, procedían de bachiller $(88.2 \%)$, eran residentes en Alicante capital $(30.5 \%)$ o Alicante provincia (30.3\%) y no trabajaban (90.0\%). De todos ellos, el 32.0\% eran <20 años, el 55.4\% tenían 20-24 años, el 8.8\% tenían 25-29 años y el 3.9\% eran $\geq 30$ años. En la Tabla 1 se muestra la tasa de respuesta por curso académico de los estudiantes de medicina de la UMH. La tasa de respuesta global fue del $55.26 \%$. 
Tabla 1. Tasa de respuesta de los estudiantes de medicina de la UMH por curso.

\begin{tabular}{|c|c|c|c|}
\hline Curso & Matriculados & Respuesta & $\mathbf{\%}$ \\
\hline Primero & 139 & 84 & 60,4 \\
\hline Segundo & 148 & 78 & 52,7 \\
\hline Tercero & 150 & 86 & 57,3 \\
\hline Cuarto & 146 & 66 & 45,2 \\
\hline Quinto & 132 & 55 & 41,7 \\
\hline Sexto & 140 & 104 & 74,3 \\
\hline Total & $\mathbf{8 5 5}$ & $\mathbf{4 7 3}$ & $\mathbf{5 5 , 3}$ \\
\hline
\end{tabular}

\subsection{Alimentación y actividad física}

En lo referente a la alimentación, el $54.2 \%$ de estudiantes presentaron una baja adherencia a la dieta mediterránea frente a un $45.8 \%$ con adherencia aceptable. Un $14.1 \%$ de estudiantes seguía una dieta especial y de estos, un $4.6 \%$ era vegetariano y un $7.0 \%$ seguía otras dietas. Fueron recogidas también otras características de la alimentación. En cuanto a la actividad física, el $11.5 \%$ no realizaba ejercicio físico, el $24.7 \%$ hacía alguna actividad física o deportiva ocasional, el $16.8 \%$ realizaba actividad física varias veces al mes y el $46.9 \%$ practicaba entrenamiento deportivo o físico varias veces a la semana.

\subsection{Consumo de tóxicos}

Se estudió el consumo de alcohol, tabaco y cannabis. El 13.5\% de estudiantes refirió no consumir nunca alcohol, el $25.6 \%$ indicó un consumo de alguna bebida alcohólica $<1$ vez al mes, el $51.1 \%$ de 2 a 4 veces al mes y el $9.7 \%$ de $\geq 2$ veces a la semana. Asimismo, el $82.9 \%$ no fumaba, el $9.0 \%$ era exfumador y el $8.1 \%$ era fumador. Finalmente, el $45.9 \%$ refirió haber consumido cannabis, marihuana o hachís alguna vez en su vida.

\subsection{Salud autopercibida y salud mental}

En la Tabla 2 se pueden observar los resultados obtenidos en relación al estado de salud autopercibida y la prevalencia de ansiedad, depresión y ansiedad o depresión de los estudiantes de medicina de la UMH. Según la EVA del EQ-5D, el 8.9\% (IC 95\% 6.8-11.8) de encuestados presentaron una salud autopercibida regular-mala-muy mala. Según la EADG, presentaron una prevalencia de probable ansiedad del 54.9\% (IC 95\% 52.8-61.7), de probable depresión del $60.9 \%$ (IC 95\% 56.7-65.8) y de probable ansiedad o depresión del $73.3 \%$ (IC 95\% 69.2-77.8).

Tabla 2. Prevalencia estimada en estudiantes de medicina de la UMH

\begin{tabular}{|c|c|c|c|c|}
\hline \multicolumn{2}{|c|}{} & n & $\mathbf{\%}$ & IC 95\% \\
\hline \multirow{2}{*}{ Salud autopercibida } & Buena-muy buena & 430 & 91,1 & $88,2-93,2$ \\
\cline { 2 - 5 } & Regular-mala-muy mala & 42 & 8,9 & $6,8-11,8$ \\
\hline \multirow{2}{*}{ Ansiedad total } & No & 212 & 45,1 & $38,3-47,2$ \\
\cline { 2 - 5 } & Probable & 258 & 54,9 & $52,8-61,7)$ \\
\hline \multirow{2}{*}{\begin{tabular}{c} 
Depresión total \\
\cline { 2 - 5 } $\begin{array}{c}\text { Ansiedad o } \\
\text { depresión }\end{array}$
\end{tabular}} & No & 174 & 39,1 & $34,2-43,3$ \\
\cline { 2 - 5 } & Probable & 271 & 60,9 & $56,7-65,8$ \\
\hline \multirow{2}{*}{ No } & 120 & 26,7 & $22,2-30,8$ \\
\hline
\end{tabular}

\subsection{Asociación de variables}

- Salud autopercibida: en la tabla 3 se muestra el modelo logístico multivariante para salud autopercibida regula-mala-muy mala. Se observó una mayor probabilidad de 
salud autopercibida regular-mala-muy mala con estar en tercer curso $(\mathrm{OR}=6.620$; $\mathrm{p}=0.003)$ y en los estudiantes que seguían una dieta vegetariana $(\mathrm{OR}=4.964 ; \mathrm{p}=0.006)$. Los estudiantes que consumían alcohol $\geq 2$ veces/semana respecto a los que nunca consumían alcohol ( $\mathrm{OR}=0.067 ; \mathrm{p}=0.022)$; los que nunca habían fumado ( $\mathrm{OR}=0.244$; $\mathrm{p}=0.021)$ y los que no añadían azúcar a algunas bebidas $(\mathrm{OR}=0.390 ; \mathrm{p}=0.031)$ se asociaron a una menor probabilidad de salud autopercibida regular-mala-muy mala.

Tabla 3. Modelo logístico multivariante para presencia de salud autopercibida.

\begin{tabular}{|c|c|c|c|c|}
\hline & & OR & IC 95\% & p-valor \\
\hline \multirow{6}{*}{ Curso académico } & Primero & 1 & & \\
\hline & Segundo & 0,902 & $0,183-4,439$ & 0,899 \\
\hline & Tercero & 6,620 & $1,933-22,675$ & $0,003^{*}$ \\
\hline & Cuarto & 1,662 & $0,374-7,388$ & 0,505 \\
\hline & Quinto & 2,450 & $0,543-11,064$ & 0,244 \\
\hline & Sexto & 2,821 & $0,757-10,514$ & 0,122 \\
\hline \multirow{4}{*}{$\begin{array}{l}\text { ¿Con qué frecuencia } \\
\text { consume alguna } \\
\text { bebida alcohólica? }\end{array}$} & Nunca & 1 & & \\
\hline & $\leq 1 \mathrm{vez} / \mathrm{mes}$ & 0,731 & $0,262-2,042$ & 0,550 \\
\hline & $2-4$ veces $/$ mes & 0,436 & $0,160-1,189$ & 0,105 \\
\hline & $\geq 2$ veces / semana & 0,067 & $0,007-0,675$ & $0,022^{*}$ \\
\hline \multirow{3}{*}{$\begin{array}{c}\text { ¿Podría decirme si } \\
\text { fuma? }\end{array}$} & Fuma & 1 & & \\
\hline & Exfumador & 0,861 & $0,212-3,492$ & 0,834 \\
\hline & Nunca & 0,244 & $0,074-0,810$ & $0,021^{*}$ \\
\hline $\begin{array}{l}\text { ¿Le añades azúcar a } \\
\text { algunas bebidas? }\end{array}$ & No & 0,390 & $0,166-0,918$ & $0,031^{*}$ \\
\hline Dieta vegetariana & Sí & 4,964 & $1,565-15,751$ & $0,006^{*}$ \\
\hline
\end{tabular}

${ }^{*} \mathrm{p}<0,05$

- Ansiedad: en la tabla 4 se muestra el modelo logístico multivariante para ansiedad. Se observó una mayor probabilidad de ansiedad en las mujeres ( $\mathrm{OR}=2.470 ; \mathrm{p}<0.001)$. Los estudiantes que no procuraban tomar mucha fibra $(\mathrm{OR}=0.527 ; \mathrm{p}=0.003)$ presentaron una menor probabilidad de ansiedad.

Tabla 4. Modelo logístico multivariante para presencia de ansiedad

\begin{tabular}{|c|c|c|c|c|}
\hline \multicolumn{2}{|c|}{} & OR & IC 95\% & p-valor \\
\hline \multirow{4}{*}{ Edad } & $<20$ años & 1 & & \\
\cline { 2 - 5 } & $20-24$ años & 1,017 & $0,662-1,563$ & 0,939 \\
\cline { 2 - 5 } & $25-29$ años & 1,628 & $0,777-3,411$ & 0,197 \\
\cline { 2 - 5 } & $\geq 30$ años & 1,368 & $0,484-3,866$ & 0,555 \\
\hline \multirow{2}{*}{ Sexo } & Hombre & 1 & & $<0,001^{*}$ \\
\cline { 2 - 5 } & Mujer & 2,470 & $1,631-3,740$ & $0,003^{*}$ \\
\hline $\begin{array}{c}\text { ¿Procura tomar } \\
\text { mucha fibra? }\end{array}$ & No & 0,527 & $0,347-0,800$ & \\
\hline
\end{tabular}

${ }^{*} p<0,05$

- Depresión: en la Tabla 5 se muestra el modelo logístico multivariante para depresión. Se observó una mayor probabilidad de depresión en las mujeres $(\mathrm{OR}=2.738 ; \mathrm{p}=0.001)$ y en los estudiantes que residían en la provincia de Alicante $(\mathrm{OR}=1.742 ; \mathrm{p}=0.045)$ respecto a vivir en la capital. Estar en primer curso respecto a cuarto (OR=0.364; $\mathrm{p}=0.011)$ o quinto $(\mathrm{OR}=0.306 ; \mathrm{p}=0.004)$ supuso también una mayor probabilidad de depresión. 
Tabla 5. Modelo logístico multivariante para presencia de depresión

\begin{tabular}{|c|c|c|c|c|}
\hline & & OR & IC $95 \%$ & p-valor \\
\hline \multirow{6}{*}{$\begin{array}{l}\text { Municipio de } \\
\text { residencia }\end{array}$} & Alicante capital & 1 & & \\
\hline & Alicante provincia & 1,742 & $1,012-2,999$ & $0,045^{*}$ \\
\hline & San Juan & 1,914 & $0,991-3,700$ & 0,053 \\
\hline & Elche & 1,906 & $0,966-3,763$ & 0,063 \\
\hline & Otras provincias & 1,052 & $0,242-4,579$ & 0,946 \\
\hline & Región de Murcia & 1,323 & $0,471-3,716$ & 0,595 \\
\hline \multirow{2}{*}{ Sexo } & Hombre & 1 & & \\
\hline & Mujer & 2,738 & $1,735-4,321$ & $0,001^{*}$ \\
\hline \multirow{6}{*}{ Curso académico } & Primero & 1 & & \\
\hline & Segundo & 0,723 & $0,346-1,509$ & 0,387 \\
\hline & Tercero & 1,005 & $0,478-2,114$ & 0,989 \\
\hline & Cuarto & 0,364 & $0,167-0,792$ & $0,011^{*}$ \\
\hline & Quinto & 0,306 & $0,135-0,693$ & $0,004^{*}$ \\
\hline & Sexto & 0,656 & $0,329-1,309$ & 0,232 \\
\hline \multirow{4}{*}{ Actividad física } & No hago ejercicio & 1 & & \\
\hline & Actividad ocasional & 1,137 & $0,518-2,494$ & 0,749 \\
\hline & Varias veces al mes & 1,064 & $0,461-2,458$ & 0,885 \\
\hline & Varias veces a la semana & 0,570 & $0,279-1,163$ & 0,122 \\
\hline \multirow{2}{*}{$\begin{array}{c}\text { Adherencia a la } \\
\text { dieta mediterránea }\end{array}$} & Aceptable $\geq 9$ & 1 & & \\
\hline & Baja $<9$ & 1,506 & $0,972-2,334$ & 0,067 \\
\hline \multirow{2}{*}{ Otras dietas } & No & 1 & & \\
\hline & $\mathrm{Si}$ & 2,462 & 0,977-6,201 & 0,056 \\
\hline
\end{tabular}

${ }^{*} \mathrm{p}<0,05$

- Ansiedad o depresión: en la Tabla 6 se muestra el modelo logístico multivariante para ansiedad o depresión. Se observó una mayor probabilidad de ansiedad o depresión en las mujeres $(\mathrm{OR}=3.549 ; \mathrm{p}<0.001)$. Los estudiantes que no procuraban tomar mucha fruta $(\mathrm{OR}=0.566 ; \mathrm{p}=0.021)$ y no picaban entre comidas $(\mathrm{OR}=0.533 ; \mathrm{p}=0.010)$ presentaron una menor probabilidad de ansiedad o depresión.

Tabla 6. Modelo logístico multivariante para presencia de ansiedad o depresión

\begin{tabular}{|c|c|c|c|c|}
\hline \multicolumn{2}{|c|}{} & OR & IC 95\% & p-valor \\
\hline \multirow{4}{*}{ Edad } & $<20$ años & 1 & & \\
\cline { 2 - 5 } & $20-24$ años & 0,590 & $0,346-1,005$ & 0,052 \\
\cline { 2 - 5 } & $25-29$ años & 0,820 & $0,346-1,946$ & 0,653 \\
\cline { 2 - 5 } & $\geq 30$ años & 1,000 & $0,252-3,964$ & 1,000 \\
\hline \multirow{2}{*}{ Sexo } & Hombre & 1 & & $<0,001^{*}$ \\
\cline { 2 - 5 } & Mujer & 3,549 & $2,246-5,608$ & $0,021^{*}$ \\
\hline $\begin{array}{c}\text { ¿Procura tomar } \\
\text { mucha fruta }\end{array}$ & No & 0,566 & $0,348-0,920$ & $0,010^{*}$ \\
\hline $\begin{array}{c}\text { ¿Sueles comer } \\
\text { entre comidas? }\end{array}$ & No & 0,533 & $0,330-0,863$ & \\
\hline
\end{tabular}

${ }^{*} \mathrm{p}<0,05$ 


\section{Discusión}

\subsection{Salud autopercibida}

La salud autopercibida regular-mala-muy mala se asoció con estar en tercer curso académico, así como en los estudiantes que seguían una dieta vegetariana. Esto podría entenderse en aquellos estudiantes que ya presentaban una peor salud autopercibida y procuran cambiar su estilo de vida para mejorar su salud. En cuanto al consumo de tóxicos, se observó una mayor prevalencia de salud autopercibida buena-muy buena con el consumo de alcohol $\geq 2$ veces/semana y en los estudiantes no fumadores. Sin embargo, la relación entre el consumo de tóxicos y la salud autopercibida no está claramente establecida. Un ejemplo de ello es un estudio de 2018 en población adulta que estimó que los individuos no fumadores presentaron una peor salud autopercibida, mientras que el consumo de alcohol no se relacionó de forma estadísticamente significativa (17).

\subsection{Depresión y ansiedad}

Nuestro estudio estimó un aumento de la probabilidad de depresión y ansiedad en mujeres, al igual que en población general. Además, se estimó una mayor probabilidad de depresión entre los estudiantes que viven en pueblos de la provincia de Alicante respecto a vivir en Alicante capital. Resultados similares se observaron en un estudio polaco de 2012 que detectó una mayor probabilidad de depresión en jóvenes que viven en zonas rurales respecto a los que viven en zonas urbanas (18).

En cuanto al curso académico, se observó un aumento de probable depresión y de salud autopercibida regular-mala-muy mala entre los estudiantes de tercer curso, momento en el que comienzan las enseñanzas clínicas y la formación hospitalaria. Estos resultados fueron semejantes a los obtenidos en un estudio brasileño de 2010 sobre la calidad de vida de los estudiantes de medicina, donde el curso académico fue un determinante del impacto mental y físico negativo entre los estudiantes de tercer curso académico y posterior (19). Esto podría deberse al primer contacto con los pacientes, donde los estudiantes pasan por experiencias emocionales complicadas, o a la propia calidad de las prácticas hospitalarias. Además se estimó una alta prevalencia de probable depresión en estudiantes de primer curso respecto a cuarto y quinto que podría deberse al desafío que supone pasar de secundaria a la universidad, siendo un periodo de adaptación importante en la vida personal del estudiante. En un reciente estudio llevado a cabo en España (20) también se relacionó proporcionalmente el número de años estudiando medicina con la probabilidad de sufrir burnout. A mayor número de años de carrera, mayor puntuación en todas las escalas de burnout que miden despersonalización, agotamiento y rendimiento académico. En relación al cuestionario EADG, se observó discrepancia en los puntos de corte establecidos en diferentes estudios. En un trabajo realizado en la universidad de Murcia (21) utilizaron los mismos puntos de corte que en nuestro trabajo, obteniendo resultados similares en cuanto a prevalencia de ansiedad. Sin embargo, en otro trabajo llevado a cabo en estudiantes de medicina de una universidad peruana (22), al utilizar diferentes puntos de corte, se obtuvieron resultados inferiores en cuanto a prevalencia de ansiedad y depresión. Los resultados preliminares de un estudio realizado en España (23), cuyo objetivo fue detectar la prevalencia de ansiedad, depresión, burnout y empatía en estudiantes de medicina, reflejaron una prevalencia menor tanto de ansiedad como depresión en relación a nuestro estudio. Esta diferencia podría ser debida a los diferentes test utilizados en ambos trabajos.

Respecto a la alimentación, se observó asociación entre una mayor probabilidad de ansiedad y depresión con tomar mucha fibra. Esto podría reflejar a los estudiantes con 
mayor preocupación por la imagen corporal y por no padecer sobrepeso que deciden buscar alternativas a su alimentación habitual. Respecto a esto, un estudio de 2016 demostró que la depresión es un factor de riesgo de actitudes anormales de alimentación entre los estudiantes universitarios de la India, haciendo que el individuo sea más vulnerable al desarrollo de trastornos alimentarios (24).

La limitación propia de los estudios transversales, como es la falta de una secuencia temporal, en este caso no es relevante, ya que nuestro objetivo fue estimar prevalencias y los diferentes factores de riesgo. Además, este estudio presenta la ventaja de poder realizarse en un corto periodo de tiempo y ser el primer paso para la realización de estudios analíticos prospectivos. Por otro lado, se utilizaron solo cuestionarios validados y se obtuvo una tasa de respuesta $>50 \%$, lo que supone un buen indicador. Podrían surgir problemas al comparar la prevalencia de probable ansiedad y depresión con otros estudios transversales que utilicen diferentes escalas. Sin embargo, es importante destacar que la EADG es un cuestionario validado para screening con buenos indicadores de sensibilidad $(83 \%)$ y especificidad (82\%).

\section{Conclusiones}

- En los estudiantes de medicina de la UMH, se estimó una prevalencia de probable ansiedad del $54.9 \%$, de probable depresión del $60.9 \%$ y de probable ansiedad o depresión del $73.3 \%$

- Diversos factores como ser mujer, el curso académico, el tipo de alimentación y el consumo de tóxicos se han asociado de forma estadísticamente significativa.

- Parecen existir patrones clínicos de ansiedad y depresión que deberían ser estudiados en profundidad y tenidos en cuenta como la relación de ansiedad con el inicio de las prácticas clínicas y el comienzo de primer curso académico, así como una peor salud autopercibida en alumnos de tercer curso relacionados con el comienzo de las prácticas hospitalarias y en aquellos que siguen una dieta vegetariana con la intención de cambiar el estilo de vida.

Contribuciones de los autores: Iván Gutiérrez Pastor y Aarón Gutiérrez Pastor concibieron y diseñaron este estudio. Todas las personas firmantes participaron en la recogida de datos. José Antonio Quesada, Rauf Nouni García y Concepción Carratalá Munuera se encargaron del análisis y la interpretación de los datos. Todas las personas firmantes participaron en la revisión crítica del manuscrito con importantes contribuciones individuales y aprobaron la versión final para su publicación.

Financiación: No ha habido financiación

Declaración de conflicto de interés: Los autores declaran no tener ningún conflicto de intereses.

\section{Referencias}

1. Rodríguez-Martin A, Santi Cano MJ, Jiménez-Rodríguez A, Novalbos-Ruiz JP. Educando con el ejemplo. Alimentación y actividad física en los futuros promotores de salud. Rev Esp Nutr Comunitaria. 2019; 25(4). https://www.renc.es/imagenes/auxiliar/files/NUTRICION_COMUNITARIA_4-2019_articulo_6.pdf

2. World Health Organization (2019). Unhealty diets \& physical inactivity. Consultado el 19 de Diciembre de 2019. https://www.who.int/nmh/publications/fact_sheet_diet_en.pdf

3. Ortiz-Moncada R, Norte Navarro AI., Zaragoza Marti A., Fernández Sáez J, Davó Blanes MC. Do the Spanish university students follow Mediterranean dietary patterns? Nutr Hosp. 2012; 27(6):1954-9. http://doi.org/10.20960/nh.583

4. Martínez-González MA, Salas-Salvadó J, Estruch R, Corella D, Fitó M, Ros E; PREDIMED INVESTIGATORS. Benefits of the Mediterranean Diet: Insights From the PREDIMED Study. Prog Cardiovasc Dis. 2015 Jul-Aug;58(1):50-60. http://doi.org/10.1016/j.pcad.2015.04.003

5. Navarrete-Muñoz EM, Valera-Gran D, Gonzalez-Palacios S, García de la Hera M, Gimenez-Monzo D, Torres Collado L, Vioque J. The DiSA-UMH Study: A prospective cohort study in health science students 
from Miguel Hernández University. Rev Esp Nutr Hum Diet. 2016; 20(1): 69-76. doi: 10.14306/renhyd.20.1.188

6. World Health Organization. (2019). Tabaco. Consultado el 21 de Diciembre de 2019. https://www.who.int/es/news-room/fact-sheets/detail/tobacco

7. World Health Organization(2019). Alcohol. Consultado el 21 de Diciembre de 2019. .https://www.who.int/es/news-room/fact-sheets/detail/alcohol

8. Scholz A, Navarrete-Muñoz E. M, Garcia de la Hera, M, Gimenez-Monzo D, Gonzalez-Palacios S, ValeraGran D, Torres-Collado L, Vioque J. Alcohol consumption and Mediterranean Diet adherence among health science students in Spain: the DiSA-UMH Study. Gac Sanit. 2016; 30(2): 126-132. http://doi.org/10.1016/j.gaceta.2015.10.011

9. Instituto Nacional de Estadística (2019). Consumo de tabaco según sexo y grupo de edad. Población de 15 y más años. Consultado el 21 de Diciembre de 2019. https:/ / www.ine.es/jaxi/Tabla.htm?path=/t15/p419/a2011/p06/\&file=06017.px.

10. Roncero C, Egido A, Rodríguez-Cintas L, Pérez-Pazos J, Collazos F, Casas M. Substance Use among Medical Students: A Literature Review 1988-2013. Actas Esp Psiquiatr. 2015 May-Jun;43(3):109-21. Epub 2015 May 1. PMID: 25999158.

11. World Health Organization (2019). Global Strategy for Health for All by the Year 2000. Consultado el 22 de Diciembre de 2019. http://whqlibdoc.who.int/publications/9241800038.pdf

12. Helliwell, J. F., Layard, R., \& Sachs, J. (2012, April 30). World happiness report [2012] [R]. doi: http://dx.doi.org/10.14288/1.0053622

13. Steptoe A, Wardle J. Life skills, wealth, health, and wellbeing in later life. Proc Natl Acad Sci U S A. 2017 Apr 25;114(17):4354-4359. http://doi.org/10.1073/pnas.1616011114

14. Quek TT, Tam WW, Tran BX, Zhang M, Zhang Z, Ho CS, Ho RC. The Global Prevalence of Anxiety Among Medical Students: A Meta-Analysis. Int J Environ Res Public Health. 2019 Jul 31;16(15):2735. http://doi.org/10.3390/ijerph16152735

15. Rotenstein LS, Ramos MA, Torre M, Segal JB, Peluso MJ, Guille C, Sen S, Mata DA. Prevalence of Depression, Depressive Symptoms, and Suicidal Ideation Among Medical Students: A Systematic Review and Meta-Analysis. JAMA. 2016 Dec 6;316(21):2214-2236. http://doi.org/10.1001/jama.2016.17324.

16. Moir F, Yielder J, Sanson J, Chen Y. Depression in medical students: current insights. Adv Med Educ Pract. 2018 May 7;9:323-333. http://doi.org/10.2147/AMEP.S137384

17. Uhernik A.I, Skoko-Poljak D, Dečković-Vukres V, Jelavić M, Mihel S, Benjak T, Štefančić V, Draušnik Ž, Stevanovic R.G. Association of Poor Self-Perceived Health with Demographic, Socioeconomic and Lifestyle Factors in the Croatian Adult Population. Drus. Istraz. 2019; vol. 28 No. 2. http://doi.org/10.5559/di.28.2.03

18. Mojs E, Warchoł-Biedermann K, Głowacka MD, Strzelecki W, Ziemska B, Marcinkowski JT. Are students prone to depression and suicidal thoughts? Assessment of the risk of depression in university students from rural and urban areas. Ann Agric Environ Med. 2012; 19(4):770-4. http://dx.doi.org/10.1590/1518$\underline{8345.1592 .2878}$

19. Paro HB, Morales NM, Silva CH, Rezende CH, Pinto RM, Morales RR, Mendonça TM, Prado MM. Healthrelated quality of life of medical students. Med Educ. 2010 Mar;44(3):227-35. http://doi.org/10.1111/j.1365-2923.2009.03587.x

20. Gil-Calderón J, Alonso-Molero J, Dierssen-Sotos T, Gómez-Acebo I, Llorca J. Burnout syndrome in Spanish medical students. BMC Med Educ. 2021 Apr 22;21(1):231. http://doi.org/10.1186/s12909-021-02661-4

21. Balanza Galindo S, Morales Moreno I, Guerrero Muñoz J. Prevalencia de Ansiedad y Depresión en una Población de Estudiantes Universitarios: Factores Académicos y Sociofamiliares Asociados. Clínica y Salud [online]. 2009, vol.20, n.2, pp.177-187. ISSN 2174-0550

22. Osada J, Rojas M, Rosales C, Vega-Dienstmaier J. Sintomatología ansiosa y depresiva en estudiantes de medicina. RNP [Internet]. 22feb.2013 [citado 5may2021];73(1):15-9. Available from: https://revistas.upch.edu.pe/index.php/RNP/article/view/1651

23. Capdevilla P, García-Abajo JM, Flores-Funes D, García-Barbero M, García-Estañ J. Prevalencia de Depresión, Ansiedad, Burnout y Empatía en estudiantes de Medicina de España. Boletín de educación médica DocTutor. Publicada Nov 3, 2020-Actualizada Dic 8, 2020. 3, 2020. Consultado el 5 de Mayo de 
2021. Disponible en http://www.doctutor.es/2020/11/03/prevalencia-de-depresion-ansiedad-burnouty-empatia-en-estudiantes-de-medicina-de-espana/

24. Kar A, Bhattacharyya S. A Study on the Relation between Depression and Anxiety with Eating Disorder in Students, Politicians and Businessmen of a Suburb Region of Kolkata, India. IJHSR. 2016; 6(2): 315-322. https://www.ijhsr.org/IJHSR_Vol.6_Issue.2_Feb2016/45.pdf

(C) 2021 por los autores. Enviado para su publicación en acceso abierto bajo los términos y condiciones de la licencia Creative Commons Attribution (CC BY) (http://creativecommons.org/licenses/by/4.0/). 